\title{
Geochemical and Biochemical Factors Controlling Skeletal Nucleation and Their Impact on Coral in a Changing Ocean
}

\author{
MARTA WOLFSHORNDL ${ }^{1}$, AMY LARSEN, ALEXANDER C. \\ GAGNON $^{1 *}$ \\ ${ }^{1}$ School of Oceanography, University of Washington, Seattle, \\ WA, USA (*correspondence: gagnon@uw.edu)
}

Nucleation acts as a gatekeeper, controlling the location, timing, and even the composition of skeletal growth in both juvenile and adult coral, with implications for calcification rates in a changing ocean, coral-based paleoproxies, reef strength in the face of storms, and larval development. However, the energetics and regulation of this critical chemical process are poorly understood in coral. Indeed, the same characteristics that allow nucleation to act as a useful physiological checkpoint, namely large energy barriers coupled with high-order sensitivity to local chemistry, also imply that nucleation should be exceptionally sensitive to environmental conditions like ocean acidification. We conducted inorganic nucleation rate experiments under controlled chemical conditions to measure the sensitivity of aragonite nucleation in seawater to oversaturation, and determined that, in the range of environmentally relevant oversaturation values, nucleation is more sensitive to the chemical environment than mineral growth rate is. Using our inorganic nucleation rates and aragonite mineral growth rates from previous work, we developed a numeric model to predict how the relative sensitivities of these two modes of growth affect calcification. We found that horizontal extension of the skeleton is controlled by nucleation while vertical extension is controlled by mineral growth rate, and that horizontal extension is therefore potentially more tunable by the coral through the use of interfacial energy changes from organic matrix proteins. To test the extent to which corals have biological control over the nucleation process as well as to understand the mechanisms of how they do so, inorganic nucleation flow cell experiments were performed with the addition of matrix protein analogues to mimic the protein templates corals use in their organic matrix. These experiments quantify the limits of corals' ability to respond to the environmental pressure of ocean acidification on skeletal growth. We then tested for these nucleation effects in living coral. 